\title{
Annular Bright Field STEM Investigation of the (0001) Stacking Fault in Alumina
}

\author{
Eita Tochigi ${ }^{1}$, Teruyasu Mizoguchi ${ }^{2}$, Atsutomo Nakmura ${ }^{3}$ and Naoya Shibata ${ }^{1}$, Yuichi Ikuhara ${ }^{1,4,5}$ \\ 1. Institute of Engineering Innovation, The University of Tokyo, Tokyo, Japan. \\ 2. Institute of Industrial Science, The University of Tokyo, Tokyo, Japan. \\ 3. Department of Materials Science and Engineering, Nagoya University, Nagoya, Japan. \\ 4. Nanostructures Research Laboratory, Japan Fine Ceramics Center, Nagoya, Japan. \\ 5. Center for Elements Strategy Initiative for Structure Materials, Kyoto University, Kyoto, Japan.
}

Alumina $\left(\alpha-\mathrm{Al}_{2} \mathrm{O}_{3}\right)$ is one of widely used structural ceramics. Dislocations in alumina have been investigated for many years. The dislocations typically dissociate into some partial dislocations with a stacking fault. The stacking fault is known to be formed on the $\{11-20\}$ plane [1] or the $\{1-100\}$ plane [2]. However, stacking faults on other planes, such as the (0001) plane, are not well understood. In this study, we characterized the atomic structure of the stacking fault on the (0001) plane formed between dissociated dislocations in a low-angle grain boundary of alumina by atomic-resolution transmission electron microscopy.

An alumina bicrystal with a $(0001) /<1-100>2^{\circ}$ low-angle grain boundary was fabricated by joining a pair of a single crystal at $1500^{\circ} \mathrm{C}$ in air. A TEM sample was prepared from the bicrystal by a standard technique including argon ion milling. The dislocation structure of the grain boundary was observed by high-resolution TEM (JEM-4010, 400kV, JEOL) and scanning TEM (ARM-200F, 200kV, JEOL). For STEM observations, a probe-forming semiangle was set to be $24 \mathrm{mrad}$ and an annular detector spanning the range 12-24 mrad were used. This condition corresponds to the so called annular bright field (ABF) STEM [3].

Figure 1 shows a high-resolution TEM image of the grain boundary. Three pairs of dissociated dislocations are observed. A more detailed analysis revealed that these dislocations have the Burgers vector of $1 / 3<-1101>$ [4]. The two partial dislocations in each pair are separated by about $2.7 \mathrm{~nm}$ on the (0001) plane, suggesting the formation of a (0001) stacking fault.

The atomic structure of the (0001) stacking fault was further investigated by ABF-STEM. Figure 2 shows an ABF-STEM image of the (0001) stacking fault formed between a pair of dissociated dislocations. The atomic columns are imaged as dark contrasts. The stacking sequence of (0001) planes is found to be ...12A $32 \mathrm{~B} 12 \mathrm{C} 3 / / 1 \mathrm{~A} 21 \mathrm{~B} 31 \mathrm{C} \ldots$, where '//' indicates the position of stacking fault (Note that the stacking sequence of the perfect crystal is ...12A $32 \mathrm{~B} 12 \mathrm{C} 32 \mathrm{~A} 12 \mathrm{~B} 32 \mathrm{C} \ldots$, where 1 and 3 refer to the oxygen layer and $2 \mathrm{~A}, 2 \mathrm{~B}$, and $2 \mathrm{C}$ refer to the aluminum layer.). Interestingly, this stacking fault is formed on the anion sublattice, although only the stacking faults on the cation sublattice have been reported in alumina. In the presentation, we will discuss the structure and the formation energy of the (0001) stacking fault in detail.

\section{References:}

[1] T. E. Mitchell et al, Philos. Mag. 34 (1976), p. 441.

[2] D. G. Howitt and T. E. Mitchell, Philos. Mag. 44 (1981), p. 229. 
[3] S. D. Findlay et al., Appl. Phys. Lett. 95 (2009), 191913.

[4] E. Tochigi et al., Acta Mater. 51 (2015), p. 152.

[5] The authors acknowledge funding from the Ministry of Education, Culture, Sports, Science, and Technology in Japan (Grant Nos. 25106003, 12024046 and the project of Elements Strategy Initiative for Structural Materials) and Japan Society for the Promotion of Science (Grant Nos. 15H02290, $15 \mathrm{H} 04145$ and $15 \mathrm{~K} 20959)$.

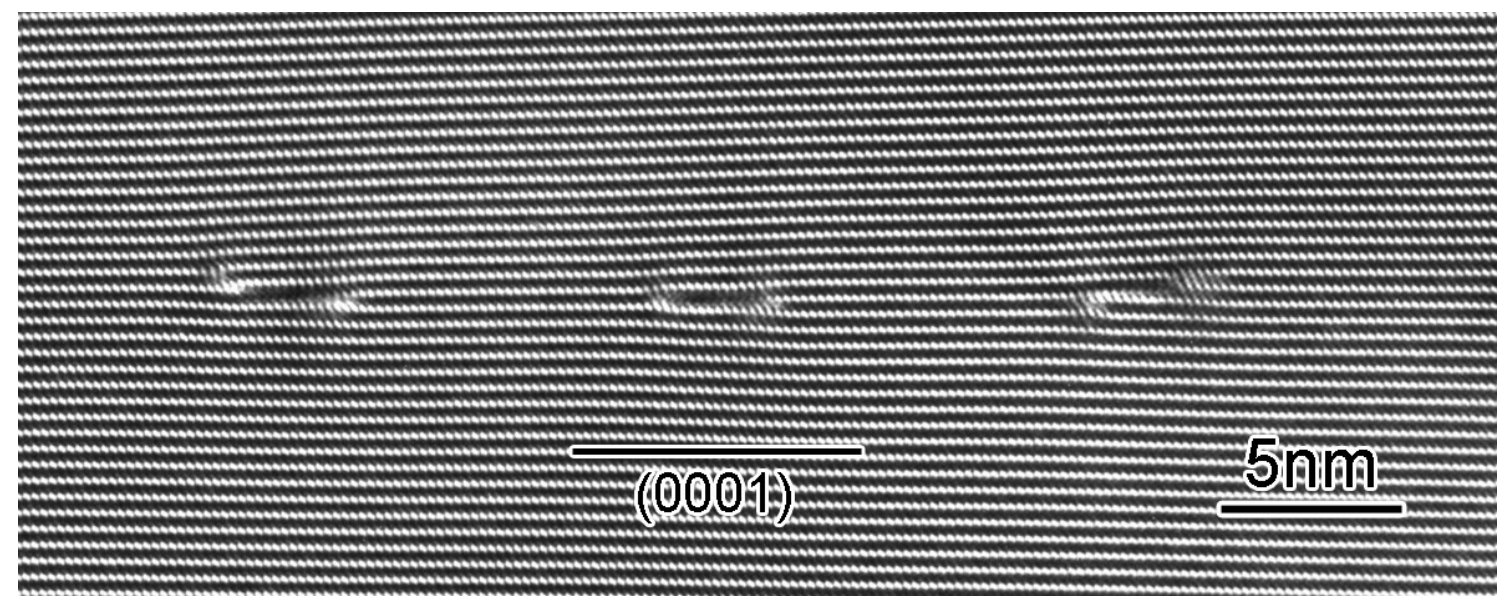

Figure 1. A high-resolution TEM image of the grain boundary viewed along the $<1-100>$ direction. Three pairs of dislocations dissociated on the (0001) plane are observed.

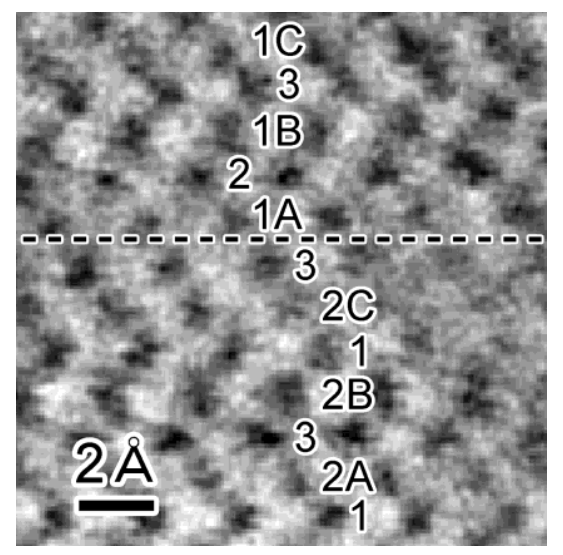

Figure 2. An ABF-STEM image of a stacking fault formed on the (0001) plane. The position of the stacking fault is indicated by the dashed line. A smoothing filter was applied to reduce visible noise. 